\title{
High Spectral Efficiency Transmission Emulation for Non-Linear Transmission Performance Estimation for High Order Modulation Formats
}

\author{
M. E. McCarthy ${ }^{(1)}$, N. Mac Suibhne, S. T. Le, P. Harper, and A. D. Ellis \\ (1) Aston Institute of Photonic Technology, Aston University, Aston Triangle, Birmingham, UK \\ $\bowtie$ m.mccarthy@aston.ac.uk
}

\begin{abstract}
We demonstrate a simple method to experimentally evaluate nonlinear transmission performance of high order modulation formats using a low number of channels and channel-like ASE. We verify it's behaviour is consistent with the AWGN model of transmission.
\end{abstract}

\section{Introduction}

As commercial system capacity approaches the fiberchannel capacity limit, as defined in references ${ }^{1-4}$, quantifying the effects of fibre nonlinearity for different transmission configurations is essential. The predominant nonlinear effect arises from the intensity dependent refractive index (Kerr effect). Channel capacity limit calculations in recent times have used the assumption that wavelength-division-multiplexed (WDM) impairments can be plausibly represented as statistically independent additive white Gaussian noise (AWGN), the variance of which may be added to that of amplified spontaneous emission (ASE). Inspired by this observation, here we propose that interfering channels can be emulated as channel-shaped ASE which is itself a good approximation of Gaussian noise ${ }^{6}$. To substantiate the proposal, we compare the transmission performance $20 \mathrm{Gbit} / \mathrm{s}$ quadrature phase shift keying (QPSK) and optical frequency division multiplex (OFDM) channels with different combinations of ASE and data modulated neighbours.

\section{High Capacity Emulation for Non-Linear Transmis-} sion

Intra-channel impairments such as chromatic dispersion and self-phase modulation effects can be compensated completely using modern digital signal processing (DSP) methods. Along with ASE, interchannel Kerr effects, which causes multiplicative noise, will be the principal sources of impairments that will limits the information capacity of an optical communication system. It induces random fluctuations in the target channel which are related to the intensity of the neighbouring WDM channels. The capacity (C) per bandwidth (B) in these conditions is bounded by ${ }^{2}$

$$
\frac{C}{B} \geq \frac{P_{s}}{P_{n}+\gamma P_{s}^{3}}
$$

where $P_{s}$ is the signal power, $P_{n}$ is the accumulated noise from amplification per span, and $\gamma$ is a scaling factor related to the nonlinearity. This bound assumes that there is negligible temporal and spatial correlation between symbols and channels ${ }^{5}$. These assumptions are valid for a dispersion uncompensated link, and they give a reasonable prediction of system performance for all high spectral efficiency modulation formats, greater accuracy is achieved for pre-dispersed signals ${ }^{8,9}$ or formats with a spectral decorrelation such as OFDM ${ }^{10}$. Such spectrally decorrelated formats tend to demonstrate Gaussian field distribution, a property which is shared by $\mathrm{ASE}^{6}$, which has negligible correlation in temporal or spectral domain. Thus we believe that it can be used to demonstrate transmission performance in a high spectral efficiency transmission configuration.

In this work, we consider a $2.5 \mathrm{THz}$ bandwidth transmission link where we use five modulated channels ( shaded red in Fig. 1) with twenty channel shaped noise neighbours spread symmetrically around the test band (green dashed boxes). This should allow us to study the nonlinear transmission performance of these five channels in a $2.5 \mathrm{THz}$ loaded system. We will use the convention that the central channel is Channel 0 and all channels are indexed from this. The central channel will have $\pm C h 1-2$ channels with the same modulation format. The outer modulated channels will only have nearest neighbours towards the centre of the spectrum with the same modulation format and will have Guassian noise neighbours towards the edge.

We study performance single carrier $20 \mathrm{Gbit} / \mathrm{s}$ QPSK which has a constant amplitude and phase across the symbol period which because of it's low bit rate, it violates the transmission decorrelation assumptions that lead to $(1)^{5}$. Also we look at the performance of OFDM QPSK with a line rate of $20 \mathrm{Gbit} / \mathrm{s}$, which satisfies assumptions in reference ${ }^{5}$ as it has complex time and spectral synthesis.

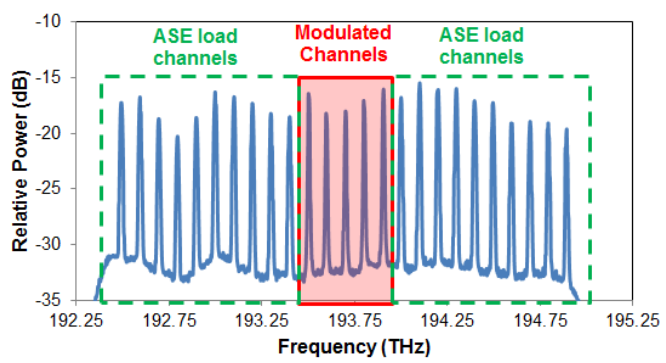

Fig. 1: Optical spectrum at $2400 \mathrm{~km}$ showing (shaded) the modulated channel and the channel-like ASE(green dashed boxes).

\section{Experimental Set-up}

The experimental set-up is shown in Fig. 2. It consisted of a laser grid of five standard DFBs on $100 \mathrm{GHz}$ grid which are substituted in turn by a $100 \mathrm{kHz}$ linewidth laser. The DFBs are located between 193.5 to 193.9 THz. The loading channels are generated using an ASE source which was spectrally shaped using a Finisar wavelength selective switch (WSS). These were set to $10 \mathrm{GHz}$ bandwidth with consistent power per channel. The twenty loading channels where spread symmetrically around the test wavelengths on a 

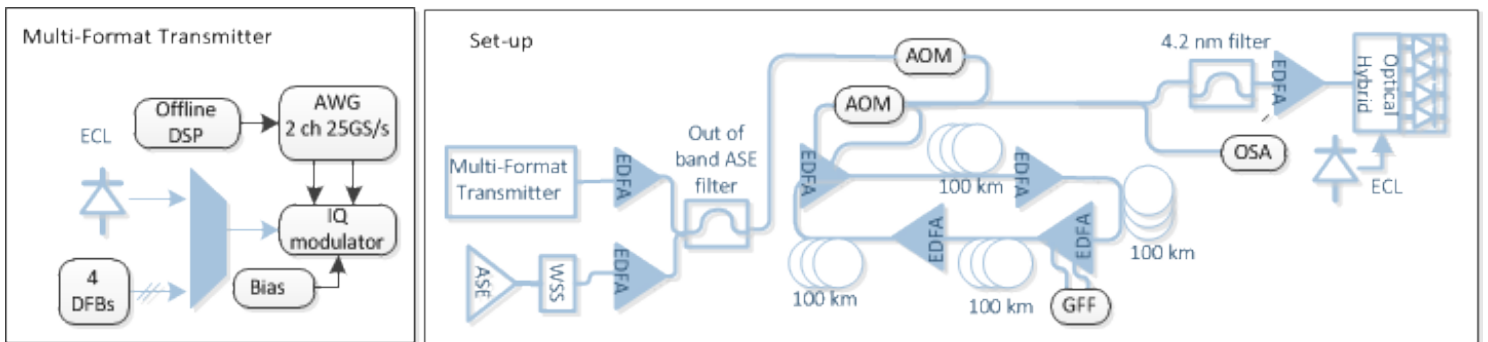

Fig. 2: Experimental set-up consisting of arbitrary waveform generator based multi-format transmitter; recirculating loop and coherent receiver;ECL: external cavity laser; DFB: distributed feedback lasers; DSP: digital signal processing; AWG arbitrary waveform generator; IQ: inphase and quadrature; WSS: wavelength selective switch; AOM: acuosto optic modulator; GFF: gain flattening filter; EDFA: Eribium doped fibre amplifier; OSA: optical spectrum analyser.

$100 \mathrm{GHz}$ grid so that the bandwidth of the transmission was $2.5 \mathrm{THz}$. Separate EDFAs were used in the transmitter for the noise loading and channels to conform to the power handling limitations of the transmit components and maintain a good transmitter optical to signal noise ratio (OSNR) and the signals was combined using a $3 d B$ coupler after amplification. $A$ wideband filter was used to remove out of band ASE noise at the transmitter.The transmission path is a acousto-optic modulator(AOM) based re-circulating loop consisting of $4 \times 100 \mathrm{~km}$ spans of Sterlite $\mathrm{OH}$ LITE (E), which including splice and patch losses, have 18.9 to $19.5 \mathrm{~dB}$ insertion loss. The amplifiers have a $6.5 \mathrm{~dB}$ noise figure under these minimum gain conditions. The GGF was optimised for a transmission distance of $2400 \mathrm{~km}$. The multi-format transmitter consisted of a laser (100 kHz linewidth); an in-phase and quadrature $(\mathrm{IQ})$ modulator biased at null point and $90^{\circ}$ offset; and a 2 channel $25 \mathrm{GS} / \mathrm{s}$ arbitrary waveform generator (AWG) programmed with either an OFDM or QPSK signal. The OFDM signal (400 symbols each of 20.48 ns length, $2 \%$ cyclic prefix) encoded with QPSK modulation format was generated offline in MATLAB using an IFFT size of 512, where 210 subcarriers were filled with data and the remainder zeros giving a line rate of $20 \mathrm{~Gb} / \mathrm{s}(18.2 \mathrm{~Gb} / \mathrm{s}$ after cyclic prefix and FEC overhead removed). The QPSK was generated with two independent PRBS streams of length $2^{15}-1$ for the IQ components. Two samples per bit were used to generate the drive signal resulting in 10 Gbaud QPSK signal. These two formats where chosen as they provide comparable data rates. At the receiver, signal is broadly pre-filtered using a flat topped programmable filter set to $4.2 \mathrm{~nm}$ and detected using a polarization diverse coherent receiver. The electrical received signals were then sampled by a realtime oscilloscope at $80 \mathrm{GS} / \mathrm{s}$ and $25 \mathrm{GHz}$ bandwidth. The local oscillator (100 kHz linewidth) was amplified to $16 \mathrm{dBm}$.

Both signals were processed offline using DSP. The single carrier QPSK DSP included signal normalization, low pass filtering, down sampling, static dispersion compensation, clock recovery, polarization de-multiplexing (constant modulus algorithm) and phase recovery. The OFDM DSP at the receiver included chromatic dispersion compensation, channel estimation and equalization with the assistance of initial training sequence (2 training symbols every 100 symbols) using zero forcing estimation, common phase error compensation with pilot subcarriers (8

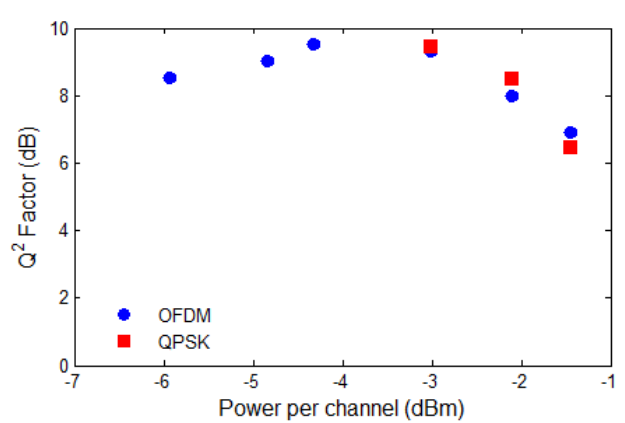

Fig. 3: Optimum Launch Power for OFDM and QPSK at 3200 $\mathrm{km}$. Note that this is averaged across all channels - hence why QPSK performance appears depressed.

pilots every OFDM symbol) and symbol detection. For both modulation formats, system performance is evaluated using the $\mathrm{Q}$-factor derived directly from the error counting using $\simeq 1 \times 10^{6}$ bits.

\section{Results and Discussion}

The optimum launch power for each of the modulation formats was identified and is shown in Fig. 3 . While the optimum launch power for the OFDM signal was easily identified, it was observed that the $Q^{2}$ factor across the 5 QPSK channels performance was uneven, with the outer channels appearing significantly degraded with respect to the centre channel. To accommodate this strong variation the signal power was set to give the optimum $\bar{Q}^{2}$. At this launch power, the centre channel was below its optimum and the edge channels were above theirs. The mean $\bar{Q}^{2}$ itself was also dominated by the four degraded channels, thus although the central QPSK channel outperformed the OFDM signals, the overall average performance of the QPSK signal was worse. This was found to be higher than expected due to lower than assumed transmitter OSNR (attributed to power limitations of WSS and single moudulator ) and noise figure of the loop amplifiers. .

The $Q^{2}$ factor for QPSK and OFDM after $2400 \mathrm{~km}$ are shown in Fig. 4 at $-3 d B m$ and $-4 d B m$ respectively. It can be seen that the central $(193.7 \mathrm{THz})$ chanel for QPSK performs 1.6 dB greater than 193.5 THz and $193.9 \mathrm{THz}$ channels which are neighbouring the noise channels. While for the OFDM, the $Q^{2}$ factor remains constant over the 5 channels under test. The constellation diagrams for both modulation formats are shown in Fig. 5

To understand this we have to consider the temporal correlation of the QPSK signal with respect to its neighbours. Due to its low bit rate, the QPSK 


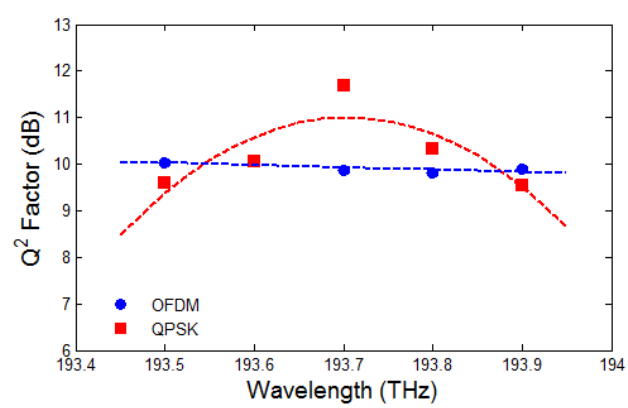

Fig. 4: $Q^{2}$ Factor for OFDM and QPSK after $2400 \mathrm{~km}$ with simple data fit

signal will maintain long term temporal correlation for much of the transmission length. Due to the effect of chromatic dispersion, this will result in adjacent symbols in the channel under test interacting with almost the same subset of interfering pulses from the neighbouring channels ${ }^{7}$. Thus continuous symbols have accumulating information about the correlated nonlinearity they have experienced. This will result in correlated nonlinear phase noise. Methods to completely compensate intra-channel phase noise is an open issue but DSP does cancel correlated nonlinear phase noise as part of normal detection. The DSP used here has not been optimised for this but performs some compensation proportional to the amount of correlated intra-channel nonlinearity. Thus for the central channel ( $\mathrm{Ch0} / 193.7 \mathrm{THz}$ ) with $\pm 1 \mathrm{Ch}$ and \pm 2 Ch QPSK neighbouring channels with simple modulation format has less residual nonlinear phase noise after DSP compared to the channels at $193.5 \mathrm{THz}$ and $193.9 \mathrm{THz}$ which have QPSK neighbours in one direction but ASE neighbours in the other.
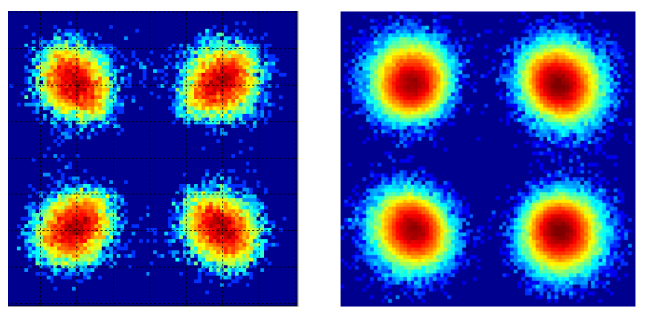

(a) QPSK Constellation (b) OFDM Constellation Diagram Diagram

Fig. 5: Example Constellation Diagrams for single-carrier QPSK and OFDM QPSK at $2400 \mathrm{~km}$

In contrast, the OFDM which has a complex synthesis sees the channel-like noise neighbours as having a similar nonlinear phase noise influence as like neighbours so has uniform performance. For the OFDM signal the structure of the transmitted signals, with many phase decorrelated subcarriers within the velocity matching bandwidth of the fibre, would be expected to generates un-correlated nonlinear phase noise. This, combined with a symbol length longer than any possible noise correlation period essentially means that the nonlinear phase noise remains after conventional OFDM DSP. This nonlinear noise thus closely resembles that generated by ASE, resulting in the uniform $Q^{2}$ factor independent of channel environment observed in Fig. 4.

\section{Conclusion}

In this paper, we have demonstrated that for OFDM signals, where there is negligible spectral correlation at the transmitter and little opportunity to exploit temporal correlation due to dispersion effects, channel emulation due to noise loading gives an accurate measure of system performance. This will enable such systems to be designed and installed using simple spectrally shaped ASE loading channels to predict future performance. For simpler signals, such as QPSK, temporal correlation allows DSP to automatically compensate for nonlinear phase noise, implying that ASE loading channels produce pessimistic results. However, despite reduced accuracy, we anticipate that QPSK channels which are correctly decoded in the presence of ASE loading channels will also decode correctly when such channels are replaced with other OFDM data carry channels.

Finally, we observe that these results, in particular Fig. 4, independently corroborate the assumptions and models in recent analyses of nonlinear capacity limits ${ }^{4,7}$. In particular, it has been proposed that when nonlinear phase noise is correlated, as in the case for the central QPSK channel here, the statistics of the nonlinear noise is modified and DSP may be used to compensate for the nonlinear phase noise components.

\section{Acknowledgment}

This work was partly funded by the European Communities 7th Framework Programme FP/2007-2013 grants 318415 (FOX-C) and 258033 (MOD-GAP), and the EPSRC grant EP/J017582/1 (UNLOC). The authors would like to thank Dr. Arvind Kumar Mishra and Dr Badri Gomatam from Sterlite Technologies for the loan of the Sterlite OH-LITE (E) Fibre used in this experiment; and Finisar Corporation for their support.

\section{References}

1 R.-J. Essiambre, et al., "Capacity limits of optical fiber networks" JLT. 28, 662 (2010).

2 A. D. Ellis,et al., "Approaching the non-linear Shannon limit" JLT. 28,423 (2010).

3 G. Bosco et al., "Analytical results on channel capacity in uncompensated optical links with coherent detection" Opt. Express 19, B440 (2011).

4 A. Mecozzi \& R.-J. Essiambre, "Nonlinear Shannon limit in pseudolinear coherent systems" JLT 30,2011 (2012).

5 P. Mitra et al., "Nonlinear limits to the information capacity of optical fibre communications" Nature, 411, 1027 (2001).

6 W S Wong, et al., "Photon statistics of amplified spontaneous emission noise in a 10-Gbit/s optically preamplified direct-detection receiver" Optics Letter 23,1832(1998).

7 R Dar, et al., "Properties of nonlinear noise in long, dispersion-uncompensated fiber links" Optics Letter 30,398(2014).

8 D Rafique, et al., "Various nonlinearity mitigation techniques employing optical and electronic approaches" PTL 23,1838(2011).

9 R. Cigliutti, et al., "Ultra-long-haul transmission of $16 \times 112 \mathrm{~Gb} / \mathrm{s}$ spectrally-engineered DAC-generated Nyquist-WDM PM-16QAM channels with $1.05 \mathrm{x}$ (symbol-rate) frequency spacing" OTh3A-3, OFC (2012).

10 L B.Du,et al., "Improved nonlinearity precompensation for long-haul high-data-rate transmission using coherent optical OFDM." Opt. Express 16,19920(2008). 